\title{
SwSim: discovering semantic similarity association in semantic web
}

\begin{abstract}
Similarity is an important and fundamental concept in AI and many other fields. In different applications, users need to discover the relations between objects and find the level of semantic similarity between them. (I.e. find two similar papers or two similar events). In order to answer these types of complex queries, discovering semantic similarity association is one of the important steps.

The semantic web describes the resources/entities and its relationships in machine understandable way. Although semantic web technologies define relations between objects but discovering the semantic similarity relation between objects is an ongoing research.

This paper presents our method (SwSim) based on semantic association concept to discover the semantic similarity in semantic web document. In this paper, we describe how the proposed method help user to answer the complex queries in semantic web.
\end{abstract}

